\title{
Development of a fluorescent probe-based recombinase polymerase amplification assay for rapid detection of Orf virus
}

\author{
Yang Yang ${ }^{1}$, Xiaodong Qin ${ }^{1}$, Guangxiang Wang ${ }^{1}$, Yuen Zhang ${ }^{1,2}$, Youjun Shang ${ }^{1}$ and Zhidong Zhang ${ }^{1 *}$
}

\begin{abstract}
Background: Orf virus (ORFV) is the causative agent of Orf (also known as contagious ecthyma or contagious papular dermatitis), a severe infectious skin disease in goats, sheep and other ruminants. The rapid detection of ORFV is of great importance in disease control and highly needed. A isothermal molecular diagnostic approach, termed recombinase polymerase amplification (RPA), is considered as an novel and rapid alternative techonology to PCR assay.

Results: In the present study, a novel fluorescent probe based on RPA assay (ORFV exo RPA assay) was developed. The developed ORFV exo RPA assay was capable of as low as 100 copies of ORFV DNA /reaction and was highly specific, with no cross-reaction with closely related viruses (capripox virus, foot-and-mouth disease virus or peste des petits ruminants virus). Further assessment with clinical samples showed that the developed ORFV exo RPA assay has good correlation with qPCR assays for detection of ORFV.
\end{abstract}

Conclusions: These results suggest that the developed ORFV exo RPA assay is suitable for rapid detection of ORFV.

Keywords: Recombinase polymerase amplification assay, RPA assay, Orf virus, Small ruminants

\section{Background}

The Orf virus (ORFV) is a prototype member of the Parapoxvirus genus within the Poxviridae family and the viral genome consists of a linear double-stranded DNA (137-139 kbp in length). ORFV is the causative agent of contagious ecthyma or contagious papular dermatitis (Orf) in goats, sheep and other ruminants, with a worldwide distribution and significant financial importance $[1,2]$. The clinical symptoms of Orf manifest as the formation of papules, vesicles and growing scabs on the lips and muzzle of infected animals $[3,4]$. Orf is usually more severe in goats than in sheep and the morbidity of the disease may reach up to $100 \%$. The mortality is usually not high but can reach up to $90 \%$ in susceptible flocks of young sheep in an epidemic situation [5, 6]. Furthermore, the disease has zoonotic potential which adds to the significance of the disease

\footnotetext{
* Correspondence: zhangzhidong@caas.cn

'State Key Laboratory of Veterinary Etiological Biology, Lanzhou Veterinary Research Institute, Chinese Academy of Agriculture Sciences, Xujiaping 1, Lanzhou 730046Gansu, China

Full list of author information is available at the end of the article
}

to public health. Therefore, the rapid detection of ORFV is of great importance in disease control and highly needed.

Although clinical signs relating to the oral mucosa and lips are indicative of Orf, a laboratory diagnosis is necessary for confirmation and epidemiological investigations. Traditional laboratory diagnostic methods include virus isolation, electron microscopy and serum neutralization [7]. However, these methods are laborious and time-consuming. Detection of serum antibody is also not effective because of the cell-mediated nature of ORFV immunity. Recently, a number of nucleic acid-based methods (such as PCR, PCRrestriction enzyme digestion and real-time PCR) have been developed for accurate and rapid diagnosis of Orf [7-10]. Among these assays, real-time quantitative PCR (qPCR) has shown to be a highly sensitive and specific assay for detection and quantification of ORFV in clinical samples and is able to differentiate from related viruses. However, qPCR assay relies on specialized and expensive thermocycling machines, as 
a result it is difficult to be used as a "pen-side" test and in endemic areas with low resources.

In recent years, a novel and rapid isothermal molecular diagnostic approach, termed recombinase polymerase amplification(RPA), has been developed as an alternative to PCR assay. The RPA technology employs three core enzymes: a recombinase, a single-stranded DNA-binding protein (SSB) and a strand-displacing polymerase. During the RPA reaction, recombinases first pairs oligonucleotide primers with homologous sequences in duplex DNA. SSB binds to displaced strands of DNA and prevents the primers from being displaced. Then the strand-displacing polymerase begins DNA synthesis at sites where the primer has bound to the target DNA. Real-time detection can be achieved by adding exonuclease III and exo probes to the reaction mixture. Importantly, RPA reaction can be performed between $37 \sim 42^{\circ}$ $\mathrm{C}$ without requirement of any sophisticated equipment. In comparison to loop-mediated isothermal amplification (LAMP) [11, 12], which requires a larger set of six primers, a higher temperature $\left(62{ }^{\circ} \mathrm{C}\right)$ and a longer run time, RPA is more rapid and simpler to run, which just needs a pair of primers [13-15], a lower temperature (37 to $42{ }^{\circ} \mathrm{C}$ ) and a shorter run time (less than $20 \mathrm{~min}$ ). Since its initial development in 2006, RPA technology has been successfully used for rapid detection of various pathogens [16-21]. In the present study, a fluorescent probe-based RPA assay has been developed and evaluated for rapid detection of ORFV. To the best of our knowledge, a RPA assay has not been developed for detection of ORFV yet. After determination of the sensitivity and specificity of the assay, clinical samples from sheep were tested and compared with results from the corresponding qPCR assay.

\section{Methods}

Virus and cells

All viruses used in this study were preserved in our laboratory: ORFV/Vaccine/CHA, ORFV/Gansu/CHA, ORFV/HB/CHA; Capripox virus CHA vaccine strain, Capripox virus/Henan/CHA; peste des petits ruminants virus (PPRV) Nigeria 75/1; foot-and-mouth disease virus (FMDV)/O/CHA, FMDV/A/CHA and FMDV/Asia 1 /CHA. A549 cells were preserved in our laboratory and cultured in minimal essential medium (MEM) containing $10 \%$ fetal bovine serum (FBS) at $37{ }^{\circ} \mathrm{C}, 5 \% \mathrm{CO}_{2}$.

\section{Sample preparation}

Twenty two field samples $(n=22)$ were collected from suspected goats of Orf and eight nasal swabs collected from eight experimentally infected sheep. Swabs were placed immediately after collection in $1 \mathrm{ml}$ phosphatebuffered saline (PBS) and stored at $-80{ }^{\circ} \mathrm{C}$ until used. To prepare ORFV-spiked tissues lysates, ORFV-free tissues samples of skin, lymphatic nodes liver, lungs, stomach and kidney ( $n=24$, three each tissue) were collected from four healthy sheep. ORFV is an epitheliotropic virus, therefore sample of skin tissue was selected. In addition, samples of other tissues were also chosen because various types of tissues are often received in the field diagnostics for differential diagnosis including nonepitheliotropic viruses such as PPRV, so it would be critical to access the compatibility of the developed RPA assay with different tissues matrix. $10 \%(\mathrm{w} / \mathrm{vol})$ tissue suspensions were then prepared by homogenizing tissue samples in PBS. Following a brief centrifugation, the homogenized tissue samples were spiked with $10^{4}$ $\mathrm{TCID}_{50}$ of ORFV/HB/CHA and stored at $-80{ }^{\circ} \mathrm{C}$ until used. To prepare ORFV infected A549 cells (human lung adenocarcinoma cells, provided by our laboratory), the A549 cells were seeded at $1 \times 10^{6}$ cells/well in 6 wellplates and cultured overnight in MEM containing $10 \%$ FBS and incubated overnight at $37{ }^{\circ} \mathrm{C}, 5 \% \mathrm{CO}_{2}$. Growth medium was removed and $100 \mu \mathrm{L}$ of ORFV/ Vaccine/CHA, ORFV/Gansu/CHA and ORFV/ HB/ $\mathrm{CHA}$ in medium with $2 \% \mathrm{FBS}$ was added into their own wells, respectively. Cells in negative control well were maintained in medium with no ORFV. After $1 \mathrm{~h}$ of adsorption at $37{ }^{\circ} \mathrm{C}$, the cells were washed gently three times with serum free medium and then maintained in MEM containing $2 \% \mathrm{FBS}$ at $37{ }^{\circ} \mathrm{C}, 5 \% \mathrm{CO}_{2}$. The cells were harvested at $12,24,36,48$ and $60 \mathrm{~h}$ after inoculation (hpi) and stored at $-80{ }^{\circ} \mathrm{C}$ until use.

\section{DNA extraction}

Total DNA was extracted from samples using high pure viral nucleic acid kit (Roche) according to the manufacturer's instructions and eluted in a final volume of $50 \mu \mathrm{L}$. Extracted DNA was stored at $-80^{\circ}$ Cuntil further use.

\section{Generation of DNA standard}

The ORFV DNA polymerase gene segments (305 bp) were synthesized by Genewiz (Suzhou, China) and cloned into a pUC57 vector, designated as pORFV/ RP1. pORFV/RP1 plasmid DNA were extracted using Plasmid Mini kit I (Promega, USA) and then measured by Nanovue (GE lifescience). The DNA copy number was calculated using the following equation [18]: DNA copy number $=\left(\mathrm{M} \times 6.02 \times 10^{23} \times 10^{-9}\right) /(\mathrm{n} \times 660)$. The DNA standard was then aliquoted and stored at $-80^{\circ}$ C until use.

\section{RPA primers and probe}

ORFV-specific RPA primers and probes, based on the highly conserved DNA polymerase gene coding sequence of ORFV, were designed according to RPA guidelines from TwistDx (Cambridge, United Kingdom). All ORFV DNA polymerase gene coding sequence genes were retrieved 
from GenBank and multiple sequence alignment of the gene sequences were manually designed based on the ORFV DNA polymerase gene recommendation by TwistDx (Cambridge, UK). All primers and probes were synthesized by Sangon Biotech (Shanghai, China). Probes were synthesized with an inverse arrangement of fluorophore (6-carboxyfluorescein [FAM]), quencher (black hole quencher 1 [BHQ-1]), spacer(tetrahydrofuran spacer[THF]) and block elongation(phosphate $[\mathrm{P}]$ ).

\section{Real-time qPCR assay}

Real-time qPCR assay was performed with SYBR • Select Master Mix on Aglient Technologies Stratagene Mx3005P thermocycler (Life technologies) as previously described [22]. The reactions were prepared as a $20 \mu \mathrm{L}$ reaction volume containing $\mathrm{SYBR}^{\circ}$ Select Master Mix (2X), the forward and reverse primers $(10 \mu \mathrm{M}, 1 \mu \mathrm{L}$ each) and $2 \mu \mathrm{L}$ of DNA template. The cycling parameters were as follows: $50{ }^{\circ} \mathrm{C}$ for $5 \mathrm{~min}, 95{ }^{\circ} \mathrm{C}$ for $10 \mathrm{~min}, 40$ cycles at $95{ }^{\circ} \mathrm{C}$ for $15 \mathrm{~s}$ and $60{ }^{\circ} \mathrm{C}$ for $1 \mathrm{~min}$. A melting curve analysis was performed using its specific melting temperature to verify the uniqueness of the amplified product. The data were analyzed using Mx3005P System software.

\section{Probe-based exo RPA assay}

Exo RPA reactions were performed in a $50 \mu \mathrm{L}$ volume using enzyme pellets from the TwistAmp exo kit (TwistDx, Cambridge, United Kingdom), which consisted of $27.5 \mu \mathrm{L}$ rehydration buffer, $2 \mu \mathrm{L}$ template DNA, $2.1 \mu \mathrm{L}$ of forward and reverse primers $(10 \mu \mathrm{M}$, BGI.tech), $0.6 \mu \mathrm{L}$ of RPA exo probe $(10 \mu \mathrm{M}$, Sangon Biotech), $11.2 \mu \mathrm{L}$ of ddH2O and $2.5 \mu \mathrm{L}$ of magnesium acetate $(280 \mathrm{mM})$. The assay was performed on Aglient Technologies Stratagene Mx3005P thermocycler (Life technologies) for 60 cycles at $40{ }^{\circ} \mathrm{C}$ for $20 \mathrm{~s}$. The reaction was completed in $20 \mathrm{~min}$. Optimal reaction conditions were defined after testing different incubation temperatures ( 39 to $42{ }^{\circ} \mathrm{C}$ ), as well as different concentrations of template $(0.5 \mu \mathrm{L}$ to $2 \mu \mathrm{L})$ and magnesium acetate $(1 \mu \mathrm{L}$ to $2.5 \mu \mathrm{L})$. During initial experiments, the original
$50 \mu \mathrm{L}$ volume of the RPA reaction was successfully reduced to $25 \mu \mathrm{L}$. Fluorescence intensity of FAM was determined every $20 \mathrm{~s}$. A sample was deemed positive if all replicates were three and a half standard deviations (3.5SD) above the background during a defined time range (i.e. after 19 to $20 \mathrm{~min}$ of amplification). A threshold time range of 0 to $4 \mathrm{~min}$ and $30 \mathrm{~s}$ was used.

\section{Statistic analysis}

Data values are provided as the mean and standard deviation (SD). Statistical analysis was performed using PRISM 5.0 software (GraphPad Software, USA).

\section{Results}

Sensitivity and specificity of ORFV exo RPA assay

Initially in order to determine the most efficient primer pair for ORFV exo RPA assay, three forward primers (F1-F3) and reverse primers (R1-R3) based on different regions of ORFV DNA polymerase gene were designed (Table 1). Nine different combination of primers (i.e. F1/ R1, F1/R2, F1/R3, F2/R1, F2/R2 F2/R3, F3/R1, F3/R2, F3/R3) were then tested with the exo probe ORFV RPA $\mathrm{P}$ on $10^{4}$ genome copies of standard DNA. The result showed that the primer set F1/R1 yielded the highest efficiency of amplification (data was shown in Additional file 1: Figure S1). Therefore, this pair of primers were employed in ORFV exo RPA assay for further validation.

To test the dynamic range of ORFV exo RPA assay, standard DNA from pORFV/RP1 was diluted 10-fold, ranging from $10^{1}-10^{6}$ genome copies per reaction and then tested by ORFV exo RPA assay. Every run was repeated 8 times. The threshold time was plotted against $\log$ (detected molecules), and a semi-log regression was calculated using PRISM 5.0 software (GraphPad Software, USA). As shown in Fig 1, the dynamic detection range of the assay spans 5 logs ranging from 6 to $2 \log$ copies per reaction, with the corresponding threshold time ranging from 2 min at $10^{6}$ copies/reaction to $10 \mathrm{~min}$ at $10^{2}$ copies/reaction. This result indicates that ORFV exo RPA assay has a wide

Table 1 RPA primers and probes designed in this study

\begin{tabular}{lll}
\hline Name & Sequence $\left(5^{\prime}{ }^{\prime} 3^{\prime}\right)$ & Genome location(U33419.1) \\
\hline ORFVRPA F1 & CTAGTAAGCTGTTCGGATCACCTTGTTCATCATG & $4465-4499$ \\
ORFV RPA F2 & TTCCGACGGACGTATGAATATGTCCATGGTGAACG & $4530-4564$ \\
ORFV RPA F3 & CGTATGAATATGTCCATGGTGAACGATGTACCAAC & $4540-4574$ \\
ORFV RPA R1 & AGCGTTCATTCAATTCATGTCTGAGGTAAACGGCA & $4702-4736$ \\
ORFV RPA R2 & GACCACGTAAAAGTGGTGTTCGAAAAACTCACAA & $4651-4685$ \\
ORFV RPA R3 & GGTAAACGGCAATGATGTTCGTGACAAAGACCACG & $4679-4713$ \\
ORFV RPA P & AACGTATCCCATGCAGTAAAGCATAGTCCG & $4582-4631$ \\
& (FAM-dT)C(THF)C(BHQ1-dT)TATAAACTCAGGAAC-p
\end{tabular}

ORFV RPA F and R, RPA primer; ORFV RPA P, RPA Exo probe; BHQ1-dT, thymidine nucleotide carrying Black Hole Quencher 1; THF, tetrahydrofuran spacer; FAM-dT, thymidine nucleotide carrying fluorescein; $P$ (phosphate), block elongation 


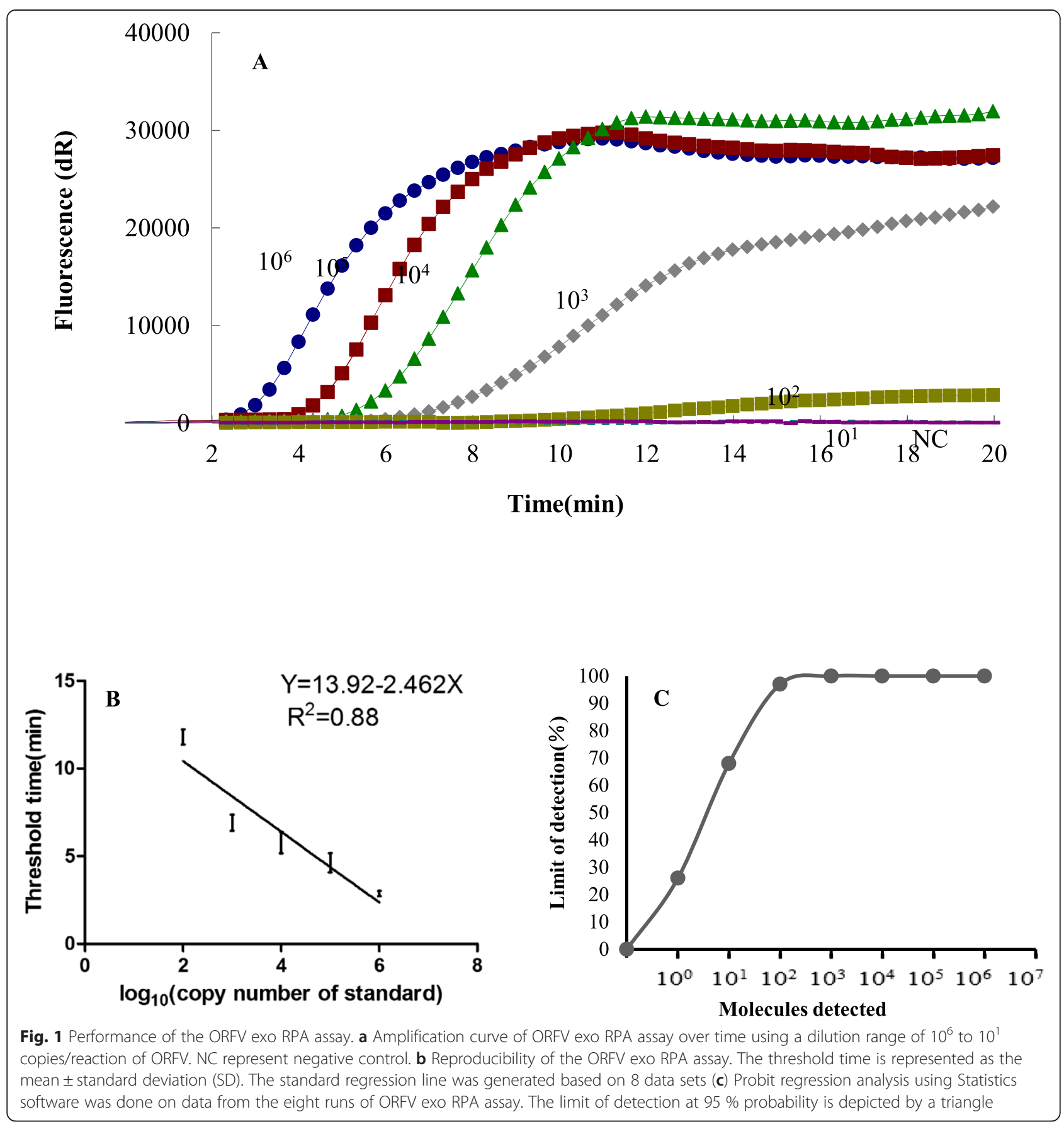

dynamic range for quantifying target DNA (Fig. 1a, b). The detection limit of ORFV exo RPA assay at $95 \%$ probability was $10^{2}$ copies per reaction (probit analysis, $\mathrm{p} \leq 0.05$ ) (Fig 1c). The specificity of ORFV exo RPA assay was determined by cross detection of other viruses that infect sheep and goat epithelium or mucus including FMDV serotypes O, A and Asia 1, PPRV and Capripox virus. No cross detections were observed and all three different ORFV strains could be detected by the developed ORFV exo RPA assay (Table 2).
To further determine the sensitivity of ORFV exo RPA assay, extracted ORFV DNA from ORFV-infected A549 cells collected at 12, 24, 36, 48 and 60 hpi were evaluated ( 2 samples each time point). The amplification of ORFV DNA in ORFV-infected A549 cells was shown successfully from as early as $12 \mathrm{hpi}$, with threshold time ( $\mathrm{min}$ ) ranging from $6.8 \pm 0.3$ at $12 \mathrm{hpi}$ to $4.3 \pm 0.4$ at $60 \mathrm{hpi}$. There was no amplification detected in the non-infected A549 cells used as negative control for this assay even through the threshold 
Table 2 Evaluation of the specificity of ORFV exo PRA assay

\begin{tabular}{|c|c|c|c|c|}
\hline Virus family & Virus specie & Virus strain & exo RPA & $\mathrm{qPCR}$ \\
\hline \multirow[t]{3}{*}{ Poxviridae } & ORFV & ORFV/Naccine/CHA & $5 \mathrm{~min}$ & $12(\mathrm{CT})$ \\
\hline & ORFV & ORFV/Gansu/CHA & $3 \min$ & $9(\mathrm{CT})$ \\
\hline & ORFV & $\mathrm{ORFV} / \mathrm{HB} / \mathrm{CHA}$ & $4 \min$ & $11(\mathrm{CT})$ \\
\hline \multirow[t]{2}{*}{ Poxviridae other than ORFV } & Capripox & Capripox virus/China Vaccine & neg & neg \\
\hline & Capripox & Capripox virus/Henan/CHA & neg & neg \\
\hline Paramyxovirinae & PPRV & Nigeria 75/1 & neg & neg \\
\hline \multirow[t]{3}{*}{ Picornaviridae } & FMDV & $\mathrm{FMDV} / \mathrm{O} / \mathrm{CHA}$ & neg & neg \\
\hline & FMDV & FMDV/A/CHA & neg & neg \\
\hline & FMDV & FMDV/Asia1/CHA & neg & neg \\
\hline
\end{tabular}

neg: negative

time (min) was greater than $30 \mathrm{~min}$. These results were $100 \%$ in agreement with these of real-time ORFV qPCR assay (a CT value ranging from $23.98 \pm 0.2$ to $14.8 \pm 0.1$ ). As a further investigation to verify sensitivity, the developed ORFV exo RPA assay was further tested using samples of ORFV-spiked tissues lysates $(n=24)$. Results showed that all the virus-spiked samples were positive for ORFV DNA, with threshold time (min) ranging from $7.1 \pm 0.4$ to $5.8 \pm 1.1$. There was no amplification detected in the non-virus-spiked samples despite threshold time being over $30 \mathrm{~min}$. These results were $100 \%$ confirmed by real-time ORFV qPCR assay (a CT value ranging from 18.4 \pm 0.8 to $21 \pm .0 .9$ ). Both assays showed the same performance on the samples above and correlation was found between values of the cycle threshold (qPCR) and threshold time (PRA) (R squared 0.64, Fig. 2).

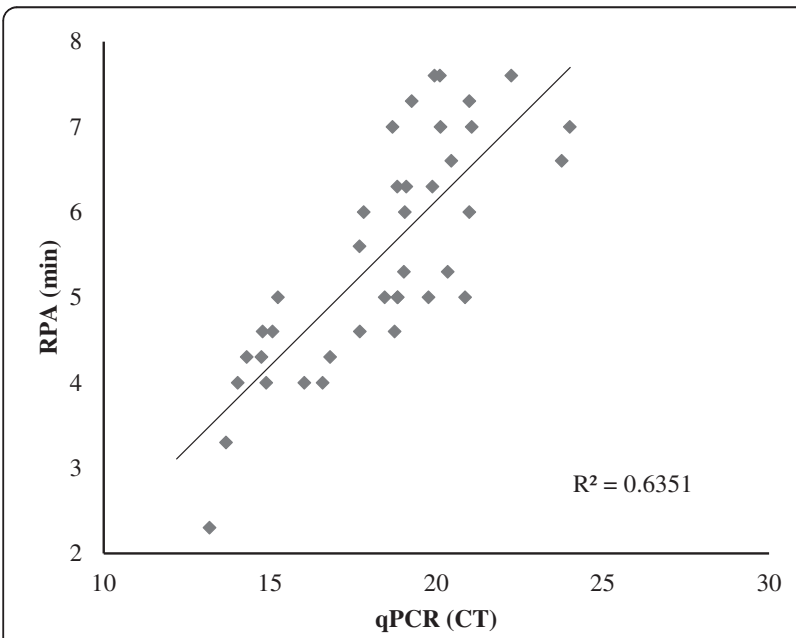

Fig. 2 Comparison between performances of ORFV exo RPA assay and real-time ORFV qPCR assay on samples of ORFV-infected cells $(n=15)$ and spiked tissues lysates $(n=24)$. Linear regression analysis of the exo RPA threshold time (y axis) and GPCR cycle threshold (CT) values ( $x$ axis) were determined by Excel software
Performance of ORFV exo RPA assay on clinical samples The practicality and efficiency of the ORFV exo RPA assay was evaluated with clinical samples and then compared with real-time ORFV qPCR assay. The clinical samples included twenty two samples $(n=22)$ collected from suspected cases of Orf, eight nasal swabs collected from experimentally infected sheep and five samples obtained from healthy goats. There was no amplification detected in samples $(n=5)$ obtained from healthy goats even through threshold time (min) exceeded $30 \mathrm{~min}$. Nasal swabs $(n=8)$ were positive for ORFV through ORFV exo RPA assay (average threshold time $=6.3 \pm 1.3$, ranging from 4.6 to 8.6). Both results above were $100 \%$ in agreement with these of the real-time ORFV qPCR (average CT value $21.3 \pm 0.3$, ranging from 16.6 to 26.2 ). Of 22 samples collected from suspected cases of the Orf, six samples were determined to be positive by ORFV exo RPA assay (threshold time ranging from 4.3 to 7.3) while eight samples were found to be positive by realtime ORFV qPCR assay (CT value ranging from 15.3 to 32.8). For the two samples found negative by ORFV exo RPA assay, the PCR assay CT value were the lowest (31.8 and 32.8, respectively). Based on a total of 35 samples examined, the sensitivity and the specificity of ORFV exo RPA assay for identification of ORFV was $86 \%$ and $100 \%$ respectively when compared to realtime ORFV qPCR (Table 3).

Table 3 Comparison of ORFV exo RPA assay with qPCR assay on clinical samples ${ }^{a}$

\begin{tabular}{lllll}
\hline & & \multicolumn{2}{l}{$\mathrm{gPCR}$} \\
\cline { 3 - 5 } & & Positive & Negative & \\
\hline RPA & Positive & 14 & 0 & 14 \\
& Negative & 2 & 19 & 21 \\
& & 16 & 19 & 35 \\
\hline
\end{tabular}

${ }^{\text {a }}$ Samples include twenty two samples collected from suspected cases of the orf, eight nasal swabs collected from experimentally infected sheep and five samples obtained from healthy goats. All samples either ORFV or no viral DNAs detected 


\section{Discussion}

In the present study, a novel fluorescent probe-based RPA assay (ORFV exo RPA assay) was developed and evaluated for detection of ORFV. We demonstrated that the developed ORFV exo RPA assay could detect ORFV at a copy as low as 100 genome copies/reaction, furthermore the results have demonstrated that the developed ORFV RPA exo assay only specifically detects ORFV.

Our data from ORFV-infected A549 cell line has shown that ORFV DNA levels increases over time after inoculation, which indicates the potential capability for quantification of the virus in samples and also that A549 cell line could be a proper cell line for ORFV replication and propagation. No amplification was observed in nonORFV infected cells/homogenizing tissue samples, this indicates the high specificity of ORFV exo RPA assay in this study. The apparent sensitivity of ORFV exo RPA assay for identification of ORFV in clinical samples was found to be $86 \%$ when compared to real-time ORFV qPCR assay. We believe that this is due to the slightly lower sensitivity of ORFV exo RPA assay and the fact that those two samples were shown to be only weakly positive by real-time ORFV qPCR (a Ct value $>31$ ). The $\mathrm{Ct}$ value of such ORFV qPCR positive/ORFV exo RPA negative samples were lower than that of the ORFV qPCR-positive/ORFV exo RPA positive samples. The results taken together indicate that the sensitivity and specificity of ORFV exo RPA assay, using F1/R1 primer set based on the region of ORFV DNA polymerase gene sequences, is comparable to real-time ORFV qPCR assay for detection of ORFV. A recent study showed that the sensitivity of RPA remains slightly lower than that of LAMP assay [23]. In comparison to RT-PCR and LAMP assays, RPA is a relatively novel and less evaluated technology. It is participated that its sensitivity could be further improved as the technology develops and the reaction conditions including RPA primers are fully optimized and evaluated. It is worth mentioning that maximal running time the developed ORFV exo RPA assay required is $30 \mathrm{~min}$, regardless of the viral concentration present in samples as long as it is above the detection limit (i.e. $>100$ copies per reaction).

In comparison, to the loop-mediated isothermal amplification (LAMP) technology, which requires a larger set of six primers, a higher temperature $\left(62{ }^{\circ} \mathrm{C}\right)$ and a longer run time, the developed ORFV exo RPA assay is simpler and more rapid. The result can be obtained in less than $20 \mathrm{~min}$ and a lower reaction temperature of $37 \sim 39{ }^{\circ} \mathrm{C}$ is needed, which is an advantage with regard to miniaturization and integration into pen-side tests. In addition, it has high specificity due to the fact that RPA uses specific detection probes, like real-time PCR assay does, while LAMP uses nonspecific intercalating fluorophores [18, 24, 25]. Unlike
LAMP assay,the specificity of the real-time fluorescent probe-based RPA assay based on TwistAmp exo kit, which was used in this study, cannot be checked in agarose gel, because the exonuclease present in the reaction mixture digests most of the amplified product once amplification has ceased. However, amplicons can be checked in agarose gel when TwistAmp basic kit or TwistAmp nfo kit in which the exonuclease is not included, is used, as we demonstrated with a specific band of amplified ORFV product in the gel electrophoresis based on TwistAmp basic kit (Additional file 2: Figure S2). Therefore, RPA has good flexibility in adaption into various detection systems.

\section{Conclusions}

The ORFV exo RPA assay described is sensitive and specific for rapid detection of ORFV within less than $30 \mathrm{~min}$. The results are encouraging but the assay must be validated by analysis of a larger number of samples from animals infected with ORFV and with different strains before such an assay can be considered for routine diagnostic use.

\section{Additional files}

\begin{abstract}
Additional file 1: Figure S1. Three forward primes (F1-F3), one probe and three reverse primers (R1-R3) were tested to select combinations yielding the highest analytical RPA sensitivity. The amplification results of nine different combination of primers with the exo probe ORFV RPA P (i.e. 1: F1/R1, 2: F2/R2, 3: F1/R2, 4: F2/R1, 5: F1/R3, 6: F2/R3, 7: F3/R1,8: F3/R2,9: F3/R3, 10: NC) were shown in the figure. Standard DNA of $10^{4}$ genome copies were used as template. (DOCX $24 \mathrm{~kb}$ )

Additional file 2: Figure S2. Analysis of the sensitivity of the recombinase polymerase amplification in agarose gel. TwistAmp basic Kit was used in this reaction. A serial dilution of the ORFV DNA standard plasmids. Positive RPA reaction products (273 bp) can be detected on a stained agarose gel (2\%) (A). NC represent negative control. (DOCX $38 \mathrm{~kb}$ )
\end{abstract}

\section{Abbreviations}

FBS: fetal bovine serum; FMDV: foot-and-mouth disease virus; LAMP: loop-mediated isothermal amplification; MEM: minimal essential medium; ORFV: orf virus; PBS: phosphate-buffered saline; PPRV: peste des petits ruminants virus; $q P C R$ : quantitative PCR; RPA: recombinase polymerase amplification.

\section{Competing interests}

The authors declare that they have no competing interests.

\section{Authors' contributions}

YY,XTQ and ZZ conceived and designed the project. YY performed the study and wrote manuscript, GXW and YJS participated in preparation of virus and samples, XTQ participated in analysis of data. XTQ, YZ and ZZ revised the manuscript. $Z Z$ is the leader of the project. All authors read and approved the final manuscript.

\section{Acknowledgements}

This work was supported by Innovation Fund of Chinese Academy of Agricultural Sciences(CAAS), China

\section{Author details}

${ }^{1}$ State Key Laboratory of Veterinary Etiological Biology, Lanzhou Veterinary Research Institute, Chinese Academy of Agriculture Sciences, Xujiaping 1, Lanzhou 730046Gansu, China. ${ }^{2}$ The Medical School, University College London, Gower Street, London WC1E 6BT, UK. 
Received: 13 October 2015 Accepted: 30 November 2015 Published online: 02 December 2015

\section{References}

1. Wang G, Shang Y, Wang Y, Tian H, Liu X. Comparison of a loop-mediated isothermal amplification for orf virus with quantitative real-time PCR. Virol J. 2013;10:138.

2. Fernandez $\mathrm{KH}$, Bream M, Ali MA, Krogmann T, Zhao H, Li Y, et al. Investigation of molluscum contagiosum virus, orf and other parapoxviruses in Iymphomatoid papulosis. J Am Acad Dermatol. 2013;68:1046-7.

3. Delhon G, Tulman ER, Afonso CL, Lu Z, de la Concha-Bermejillo A, Lehmkuhl $H D$, et al. Genomes of the parapoxviruses ORF virus and bovine papular stomatitis virus. J Virol. 2004;78:168-77.

4. Li W, Hao W, Peng Y, Duan C, Tong C, Song D, et al. Comparative genomic sequence analysis of Chinese orf virus strain NA1/11 with other parapoxviruses. Arch Virol. 2015;160:253-66.

5. Bora DP, Venkatesan G, Bhanuprakash V, Balamurugan V, Prabhu M, Siva Sankar MS, et al. TaqMan real-time PCR assay based on DNA polymerase gene for rapid detection of Orf infection. J Virol Methods. 2011;178:249-52.

6. Mondal B, Bera AK, Hosamani M, Tembhurne PA, Bandyopadhyay SK Detection of Orf virus from an outbreak in goats and its genetic relation with other parapoxviruses. Vet Res Commun. 2006;30:531-9.

7. Inoshima Y, Morooka A, Murakami K, Sentsui H. Simple preparation of parapoxvirus genome DNA for endonuclease analysis. Microbiol Immunol. 2000;44:69-72.

8. Yeruham I, Perl S, Abraham A. Orf infection in four sheep flocks. Vet J. 2000;160:74-6.

9. Haig DM. Orf virus infection and host immunity. Curr Opin Infect Dis. 2006;19:127-31.

10. Lederman ER, Austin C, Trevino I, Reynolds MG, Swanson H, Cherry B, et al. ORF virus infection in children: clinical characteristics, transmission, diagnostic methods, and future therapeutics. Pediatr Infect Dis J. 2007;26: 740-4.

11. Parida M, Sannarangaiah S, Dash PK, Rao PV, Morita K. Loop mediated isothermal amplification (LAMP): a new generation of innovative gene amplification technique; perspectives in clinical diagnosis of infectious diseases. Rev Med Virol. 2008;18:407-21.

12. Notomi T, Okayama H, Masubuchi H, Yonekawa T, Watanabe K, Amino N et al. Loop-mediated isothermal amplification of DNA. Nucleic Acids Res. 2000;28, E63.

13. Dukes JP, King DP, Alexandersen S. Novel reverse transcription loop-mediated isothermal amplification for rapid detection of foot-and-mouth disease virus. Arch Virol. 2006;151:1093-106.

14. Wei H, Zeng J, Deng C, Zheng C, Zhang X, Ma D, et al. A novel method of real-time reverse-transcription loop-mediated isotherma amplification developed for rapid and quantitative detection of human astrovirus. J Virol Methods. 2013;188:126-31.

15. Liu J, Nian QG, Li J, Hu Y, Li XF, Zhang Y, et al. Development of reversetranscription loop-mediated isothermal amplification assay for rapid detection of novel avian influenza A (H7N9) virus. BMC Microbiol. 2014;14: 271.

16. Abd El Wahed A, El-Deeb A, El-Tholoth M, Abd El Kader H, Ahmed A, Hassan S, et al. A portable reverse transcription recombinase polymerase amplification assay for rapid detection of foot-and-mouth disease virus. PLoS One. 2013;8:e71642.

17. Escadafal C, Faye O, Sall AA, Weidmann M, Strohmeier O, von Stetten F, et al. Rapid molecular assays for the detection of yellow fever virus in lowresource settings. PLoS Negl Trop Dis. 2014;8, e2730.

18. Xia X, Yu Y, Weidmann M, Pan Y, Yan S, Wang Y. Rapid detection of shrimp white spot syndrome virus by real time, isothermal recombinase polymerase amplification assay. PLoS One. 2014;9, e104667.

19. Liljander A, Yu M, O'Brien E, Heller M, Nepper JF, Weibel DB, et al. A fieldapplicable recombinase polymerase amplification assay for rapid detection of Mycoplasma capricolum subsp. capripneumoniae. J Clin Microbiol. 2015; 53(9):2810-5.

20. Abd El Wahed A, Patel P, Faye O, Thaloengsok S, Heidenreich D, Matangkasombut $P$, et al. Recombinase Polymerase Amplification Assay for Rapid Diagnostics of Dengue Infection. PLoS One. 2015;10:e0129682.

21. Boyle DS, Lehman DA, Lillis L, Peterson D, Singhal M, Armes N, et al. Rapid detection of HIV-1 proviral DNA for early infant diagnosis using recombinase polymerase amplification. MBio. 2013;4.
22. Gallina L, Dal Pozzo F, Mc Innes CJ, Cardeti G, Guercio A, Battilani M, et al. A real time PCR assay for the detection and quantification of orf virus. J Virol Methods. 2006;134:140-5.

23. Venkatesan G, Bhanuprakash V, Balamurugan V. Development and comparative evaluation of loop mediated isothermal amplification (LAMP) assay for simple visual detection of orf virus in sheep and goats. Mol Cell Probes. 2015;29:193-5.

24. Jaroenram W, Owens L. Separation of endogenous viral elements from infectious Penaeus stylirostris densovirus using recombinase polymerase amplification. Mol Cell Probes. 2014;28:284-7.

25. Yongkiettrakul S, Jaroenram W, Arunrut N, Chareanchim W, Pannengpetch $\mathrm{S}$, Suebsing $\mathrm{R}$, et al. Application of loop-mediated isothermal amplification assay combined with lateral flow dipstick for detection of Plasmodium falciparum and Plasmodium vivax. Parasitol Int. 2014;63:777-84.

\section{Submit your next manuscript to BioMed Central and we will help you at every step:}

- We accept pre-submission inquiries

- Our selector tool helps you to find the most relevant journal

- We provide round the clock customer support

- Convenient online submission

- Thorough peer review

- Inclusion in PubMed and all major indexing services

- Maximum visibility for your research

Submit your manuscript at www.biomedcentral.com/submit 